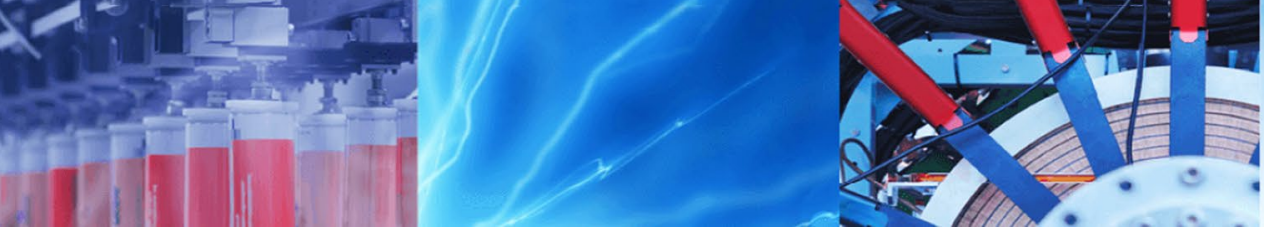

Research Article

\title{
Realization of a micro composite based pressure sensor: Its performance study for linearity, hysteresis and sensitivity
}

\author{
S. B. Subramanya ${ }^{1}$ (D) B. Pavithra ${ }^{2}$ - M. M. Nayak ${ }^{2}$-M. G. Anantha Prasad ${ }^{3}$
}

Received: 28 September 2019 / Accepted: 16 November 2019 / Published online: 30 November 2019

(c) Springer Nature Switzerland AG 2019

\begin{abstract}
The relevance of pressure sensors in daily life applications has been phenomenal in diversified disciplines such as engineering, bio-medical, automobile, aerospace etc. Making right choice of pressure sensors is crucial in any of these disciplines. Of late, the pursuit of making the pressure sensors applicable to various domains has spurred a variety of innovative activities. They are being fabricated using novel materials and unconventional fabrication methodology. Also, the advent of microelectromechanical systems has provided new horizons for a variety of realizations that led to the development of efficient and robust pressure sensors. The electromechanical characteristics of the sensing materials and the fabrication methodologies employed to realize the sensor have significant influence on performance of the sensor. This paper reports the micro composite based piezoresistive gauge pressure sensor performance study which was realized by screen printing technique. Graphite-PVC resin based dispersions are screen printed on flat, circular shaped, $1.98 \mathrm{~mm}$ thick stainless-steel diaphragm. The transducing layers are directly printed on the deforming polymer coated diaphragm so that the coupling factor is high, resulting in an efficient transfer of strain. Pressure cyclic study and its effect on linearity, hysteresis, sensitivity, stability etc. are investigated. The sensitivity is found to be $0.357 \mathrm{mV} / \mathrm{bar}$. The maximum hysteresis and non-linearity are found to be less than $0.8 \%$ FSO. A fairly satisfactory sensitivity, linearity, hysteresis and stability are accomplished at a low cost. By observing the performance characteristics of the realized pressure sensor it can be used in commercial, industrial, automotive, aerospace and strategic fields. Some of the applications could be in tire pressure sensing, air filling in petrol bunks, petrol/diesel/oil/water tankers, water supply pipelines, oil/gas explorations, hydraulic jacks and mines etc.
\end{abstract}

Keywords Pressure sensors · Piezoresistive · Screen-printing · Graphite · PVC resin · Full scale output (FSO)

\section{Introduction}

The use of pressure sensors is a widely accepted and convenient solution to monitor strain induced pressure measurements. The pursuit of developing efficient, robust and low-cost pressure sensors has spurred a variety of innovative activities in the past two decades. The piezoresistive sensors are generally more accurate and they may be considered for lower pressure applications.
They can be fabricated with fairly simple fabrication processes and sensing circuitry [1]. When piezoresistive principle of operations is employed for sensing applications, it is affected by both inter- and intra-particle resistances occurring within the sensing composite [2]. The change in resistance is likely due to either of crystal imperfections, doping concentrations, temperature, effective mass and crystallographic axes [3]. The subsequent strain sensitivity is subjected to parameters like particle conductivity,

$\triangle$ S. B. Subramanya, subramanya.sb@gmail.com | 'S D M College of Engineering and Technology (SDMCET), Dharwad, Karnataka, India. ${ }^{2}$ Centre for Nano Science and Engineering, Indian Institute of Science (IISC), Bangalore, Karnataka, India. ${ }^{3}$ Centre for Incubation, Innovation Research and Consultancy (CIIRC), Bangalore, Karnataka, India. 
percolation level and process parameters such as humidity, temperature [2] etc. The microelectronics technology employs processes like lithography, diffusion, deposition (sputtering, LPCVD, PECVD, evaporation), etching, etc. to fabricate pressure sensors. Although, they provide controlled and smooth functional layers, they turn out to be high-cost processes. They demand the availability of highend equipment, chemicals and gases that lead to, excessive impurity and toxic material wastage. Moreover, the cleanroom standards pertaining to dust free and temperature controlled environments are also of major concern. Consequently, the fabrication of pressure sensors using microelectronic processes becomes expensive and not suitable for high speed production $[4,5]$. Numerous demonstrations in the literature illustrate about the replacement of microelectronic processes by the unconventional approaches of implementing the pressure sensors. With the advent of micro and nano technologies, the recent pressure sensor design and development perspectives focus on innovative approaches of using novel strain sensing materials, in combination with newer pressure sensor fabrication techniques. A detailed see through of literature reveals that, the innovations in developing the pressure sensors are centered on the usage of different materials and fabrication technologies.

Apparently, the conventional strain sensing materials such as metal foils, semiconductors, polysilicon etc. have been replaced by polymers, resins, amorphous carbon, SW/MWCNTs, graphene and other carbon variants because of the striking advantages offered by these materials $[3,6-8]$. Also, considering the pressure sensor fabrication methodologies, a variety of novel approaches such as molding, airbrush stencil technique, spraying, hand drawing, printing, laser patterning etc., that deviate from the conventional microelectronic processes in many ways, have been attempted by various authors [5, 9-14]. Fabricating the pressure sensor devices using these techniques does not require sophisticated facilities or expertise. Also, they are proven to be flexible and robust microfabrication approaches which can be used to fabricate low-cost pressure sensors. It has enabled the sensors to be potentially applicable in different sectors such as bio-medical, automobile, aerospace, structural engineering, oceanography and many other industrial purposes.

Considering the suitability of unconventional materials in sensing applications, carbon paste derivatives are confirmed to be apt for fabrication of sensors [14]. Efficient and robust mechanical sensors can be realized using appropriately laminated printed graphite strain gauges [4]. The more direct coupling between active sensor element and measuring membrane, more stable and durable is the behavior of the sensor over time. Graphite has high electrical conductivity when compared to other carbon allotropes [10]. When graphite is embedded in polymers, the boundary resistances between particles and polymer are higher than the grains [9]. This fact allows us to assert that the charges move only through the fibers which cause resistive heating. The localized heating increases through portions of the fibers, with higher current density. A possible instability may also occur due to inefficient adhesion between filler and matrix [8]. An appropriate number of pressure cycling must be carried out to conclude about the set-in effects, non-linearity and hysteresis. The carbonbased sensors may take $50-100$ cycles until their response can be presumed stable. Graphite is inert, very less reactive to chemicals and is corrosion resistant. This fact can be utilized to produce printed carbon conductor based strain gauges that are more stable over time and more chemically resistant [10]. Electrically insulating top-coat on the printed films reduces the non-linearity and it makes the sensor response almost linear [14]. However, hysteresis effects must be considered. These strain response measurements can be carried-out either using conventional techniques or modern approaches, based on the deployment of the pressure sensor on the actual test part and environment.

Of late, the idea of printed electronics is experimented in the sensing applications and it is found to be uniquely advantageous. Cost efficiency and reduction of material wastage have aided in focusing the research on traditional printing techniques for the development of electronic devices for low operational temperatures. In this methodology, the transducing materials are printed on sensor devices using various printing techniques such as ink jet printing, screen printing, offset printing, aerosol jet printing, laser patterning $[5,11,12]$ etc. These techniques provide tremendous scope for using diversified class of substrates such as glass, acrylic, PVC, poly dimethyl siloxane (PDMS), poly methyl meta acrylate (PMMA), polyethylene terephthalate (PET, such as Mylar), polyimide (PI, such as Kapton), metal foils, paper [15] etc. Also, it is well known that electrical conduction can be achieved through formation of conductive pathways in the polymer matrix, when functional polymers are embedded on organic coatings which can be printed over many types of substrates [2]. In view of these aspects of printed electronics technology, its usage to realize pressure sensors may be considered as low-cost, high throughput approach, which can be executed in ambient conditions (temperature and pressure). Recent demonstrations illustrate that the screenprinting technology is most suitable for embedding fully 2-dimensinal strain gauge patterns. When pressure is applied on such printed coatings, a change in resistance can be observed [6]. The strain sensing characteristics of the printed strain gauges can be tuned by varying the printing parameters [5]. 
In our previous work Subramanya et al. [15], we carried out detailed experimentations and characterizations of the resistive dispersions, which are synthesized using graphite powder and letterpress ink (Supplier Data:Technical Data Sheet, InkRedible Revolution, 30.01 | 11.2007 | Web offset | Process inks, Huber Group). Screen-printed resistive dispersion strain gauges were implemented on glass substrate to carry out these experimentations. The observed features make them a good candidate for producing devices such as pressure sensors, weigh bridge load cells, displacement sensors, strain sensors, crack sensors etc. In the present work, we report about the possibility of realization and characterization of gauge pressure sensors utilizing these piezoresistive dispersions. In the current investigation, they are screen printed on a low carbon, stainless steel substrate pressure sensor device. The adhesion's reliability of dispersion onto the substrate depends on dispersion composition as well as the substrate material. For example, they adhere strongly on plastic when compared to smooth microscopic materials like glass. In the present work it is observed that, the dispersion and steel substrate exhibit good adhesion. The durability of sensing films upon the application of cyclic pressures is significantly governed by the ability of the film to expand and contract with the substrate, without suffering damage. One of the advantages of using ink in the present work is that the sensing films are highly flexible. They have high limits of elasticity (Young's modulus of ink is about $4 \mathrm{Gpa}$ ) and do not crack easily. Also, higher gage resistance of the printed films has the advantage of decreasing lead-wire effects such as circuit desensitization due to lead-wire resistance.

\section{Experimental section}

\subsection{Fabrication of pressure sensor devices}

Pressure sensor device utilized in the current work employs a low carbon content, stainless steel (AISI 304 L) and circular shaped, flat diaphragm. This specific material and shape is chosen for the diaphragm by considering, the operating pressure and, its useful properties which are desirable for substrate (diaphragm) material. It offers properties such as better mechanical strength, high fatigue life, better linearity and hysteresis response, good repeatability, better surface finish, moderate cost and bio fluidic compatibility. The chemical composition, physical and mechanical characteristics of the stainless steel diaphragm used in the present work is tabulated in Table 1.

Schematic of the pressure sensor is shown in Fig. 1a. It depicts the strain sensing films printed on the integrated diaphragm. The device consists of a stainless-steel cylindrical casing which has the diaphragm at the top side and
Table 1 Chemical composition, physical and mechanical characteristics of the diaphragm material

\begin{tabular}{ll}
\hline Composition & \\
Carbon & $: 0.06 \%$ \\
Chromium & $: 16 \%$ \\
Nickel & $: 1 \%$ \\
Molybdenum & Remaining $\%$ \\
Fe & \\
Physical characteristics & $: 7.7$ \\
Density & $: 10.1 \times 10^{-6} \mathrm{~m} / \mathrm{m}^{\circ} \mathrm{C}$ \\
Coefficient of thermal expansion & $: 2.15 \times 10^{6} \mathrm{~kg} / \mathrm{cm}^{2}$ \\
Modulus of elasticity & $: 19 \mathrm{w} \cdot \mathrm{m} / \mathrm{m}^{2}{ }^{\circ} \mathrm{C}$ \\
Thermal conductivity & \\
Mechanical characteristics & $: 122 \mathrm{~kg} / \mathrm{mm}^{2}$ \\
Tensile strength & $: 97 \mathrm{~kg} / \mathrm{mm}^{2}$ \\
Elastic limit at $0.2 \%$ & $: 16 \%$ \\
Elongation on $5 \mathrm{~d}$ & $: 90 \mathrm{to} 105 \mathrm{~h} \mathrm{Bar}(28$ to $32 \mathrm{RC})$ \\
Hardness &
\end{tabular}

Easily weldable (E.B.W) by electron beam welding or laser welding

open at the bottom. Open end at the bottom is provision for pressurizing medium (like oil, air etc.) to deflect the diaphragm. Thread at the inner periphery of the casing is used to tighten the device on the connecting adaptor for pressurization to ensure leak free operations.

Figure 2 represents the overall dimensions of the fabricated pressure sensor device. These dimensions are optimized for a pressure range of 0 to 50 Bar. Figure $3 a-c$ shows the photograph of one of the fabricated device in different views. Figure 3 a displays the top view of pressure sensor device showing the diaphragm. The cleanliness of the diaphragm surface to the microscopic level is important to ensure adequate adhesion of the insulation layer as well as printed strain sensing films. With proper surface preparation, the film properties can be reproduced. In order to free the surface from pores and other defects, it is polished with $\mathbf{4 0 0}$ grit and then, 600 grit silicon carbide emery paper (Norton Make). After this abrading operation, the surface is water sprayed and cleaned with gauge sponge to wash away the residuals. This process is repeated until the surface is free from any such residuals occurred due to abrading. After completely removing the residues, the surface is cleansed multiple times with isopropyl alcohol (IPA) for degreasing. The diaphragm is then hot air dried at $90^{\circ} \mathrm{C}$ for $30 \mathrm{~min}$ in a hot air oven (Delta Power Control) to obtain thoroughly cleansed and dried surface.

The diaphragm is insulation coated with solventthinned, epoxy-phenolic, M-Bond 610 strain gauge adhesive. It is a two-component system supplied in pre measured quantities. These were used after mixing them according to manufacturer's prescriptions. Table 2 
(a)

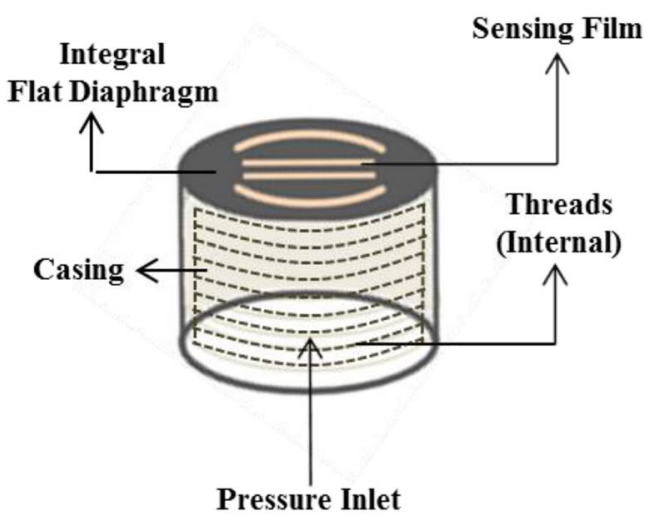

(b)

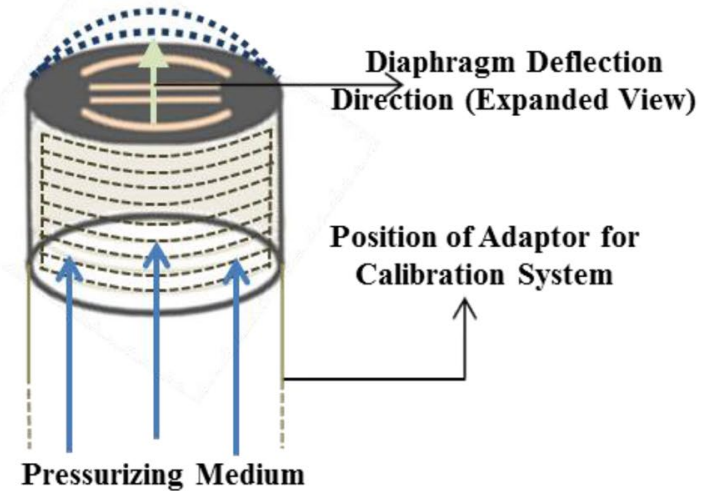

Fig. 1 Schematic of a pressure sensor, $\mathbf{b}$ pressure transduction technique

Fig. 2 Overall dimension of the device for 0 to 50 bar pressure range (all dimensions are in $\mathrm{mm})$

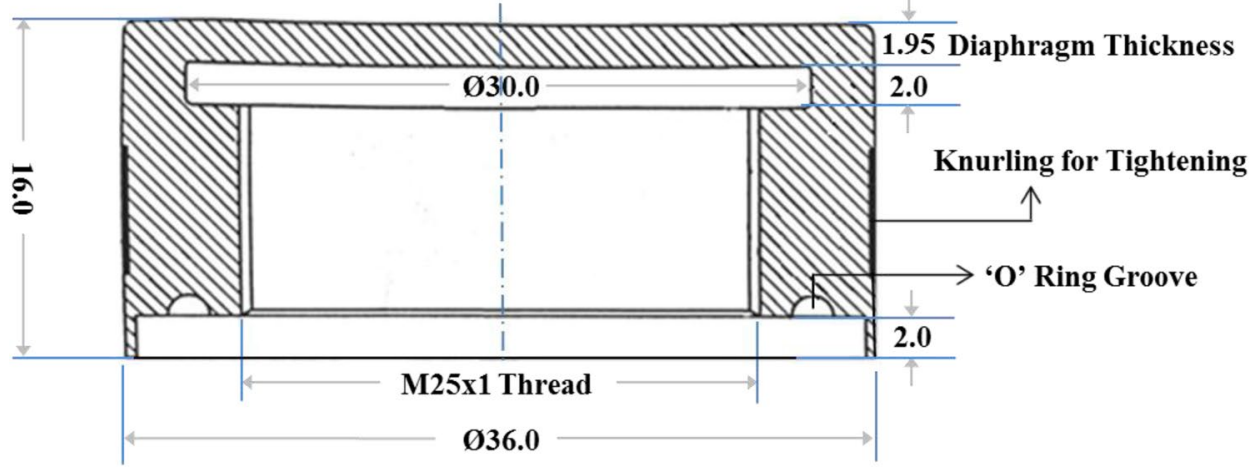

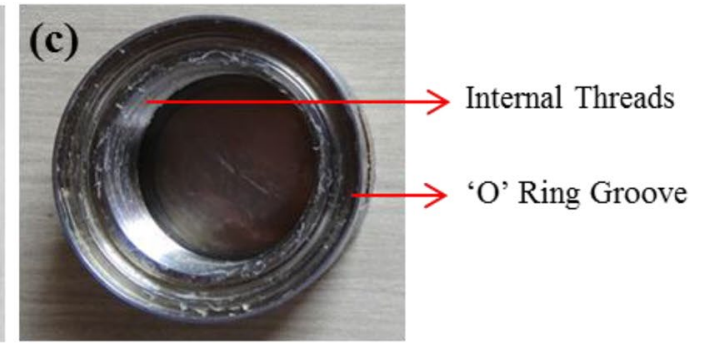

Fig. 3 a Top-view, $\mathbf{b}$ side-view, $\mathbf{c}$ bottom-view of the fabricated stainless steel pressure sensor

Table 2 Main properties of M-Bond 610 strain gauge adhesive (supplier data: instruction bulletin B-130, "strain gage installations with M-Bond 43-B, 600 and 610 adhesive systems")

\begin{tabular}{ll}
\hline Supplier & Agar scientific \\
\hline Clamping pressure & 30 to 40 psi optimum $[200$ to $275 \mathrm{kN} / \mathrm{m} 2]$ \\
Operating temperature & $-452^{\circ}$ to $+500^{\circ} \mathrm{F}\left[-269^{\circ}\right.$ to $\left.+260^{\circ} \mathrm{C}\right]$ \\
Elongation capabilities & $3 \%$ at $+75^{\circ} \mathrm{F}\left[+24^{\circ} \mathrm{C}\right] ;$ \\
Cure requirements & $2 \mathrm{~h}$ at $+400^{\circ}$ to $+450^{\circ} \mathrm{F}\left[+205^{\circ}\right.$ to \\
& $\left.+230^{\circ} \mathrm{C}\right]$ \\
Viscosity & Low viscosity, capable of glue- \\
& lines $<0.0002$ in $[0.005 \mathrm{~mm}]$. \\
\hline
\end{tabular}

summarizes some of its important properties. It is thin and hard, and, it provides void-free gluelines with minimal creep, hysteresis, and nonlinearity problems.

This coating is used to electrically isolate the strain gauge system from the metal diaphragm. The insulation resistance provided by the coating was found to be about $800 \mathrm{M} \Omega$ at $50 \mathrm{~V} \mathrm{DC}$ (measured using an insulation tester). During coating, care was taken to avoid bubble formation and achieve, uniform coat thickness. After the M-bond 610 polymer insulation layer coating, it was cured at $210^{\circ} \mathrm{C}$ for $2 \mathrm{~h}$ followed by cooling it to room temperature. 
(a)
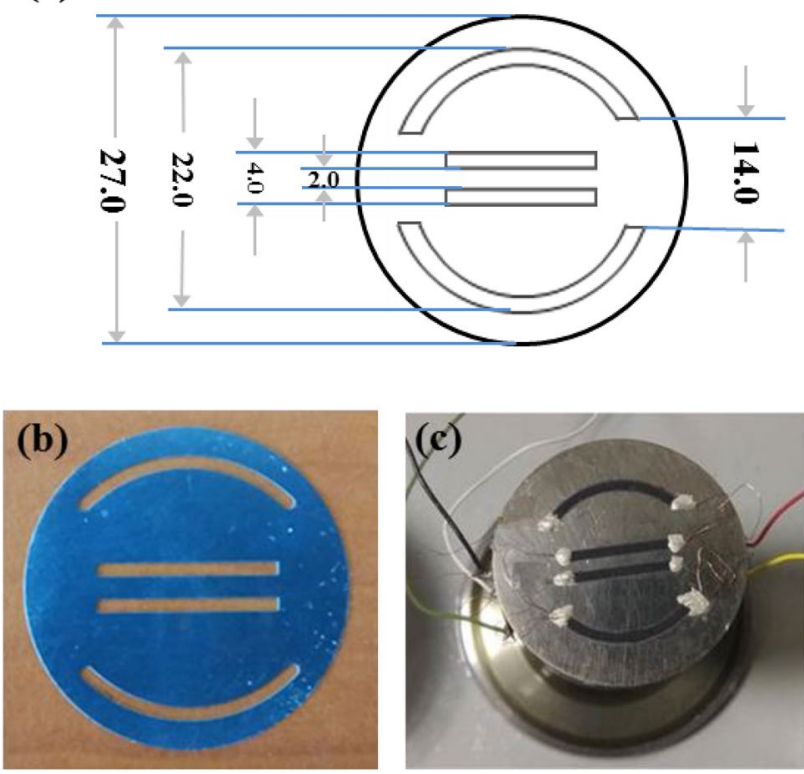

Fig. 4 a Dimensions of the mask used for screen-printing, $\mathbf{b}$ photograph of the mask, c sensing films connected in Wheatstone bridge configuration using silver paste/epoxy

Post insulation, the resistive dispersion (composed of printing-ink and graphite-powder) is screen printed on the diaphragm. Figure 4a shows the overall dimensions of the mask used for screen-printing and, Fig. $4 \mathrm{~b}$ shows the stainless-steel mask. Upon hot air blow drying (of temperature approximately $50^{\circ} \mathrm{C}$ ) the printed pattern for about $30 \mathrm{~min}$, they form elastic pressure transducing films. Figure $4 \mathrm{c}$ shows the screen-printed, strain gauge film system connected in Wheatstone bridge configuration. Double enameled copper wires of diameter $0.07 \mathrm{~mm}$ are used to interconnect the four films in Wheatstone bridge configuration through silver paste contact pads. The bridge is formed in such a way that, the gauges undergoing tensile strain are connected as one pair and the other set of gauges undergoing compressive strain are connected as another pair. A terminal strip is bonded on the sensor casing as shown in Fig. 3b. The input and output of the bridge are microsoldered to the connector strip using ERSA (Germany) make $6 \mathrm{~V}, 5$ Watt temperature controlled mini soldering station. The terminal strip is used to avoid direct connection between measuring leads and printed films, which may damage the films during calibration/measurements. Leads are attached to the Wheatstone bridge through terminal strip to perform electrical measurements as shown in Fig. 4c. The printed patterns are further passivated by parylene coating, for humidity protection and for achieving long term performance stability [15]. Figure $3 \mathrm{c}$ displays the picture of bottom-view of the pressure sensor device. The internal threads, at the inner surface of the casing can be seen in the Fig. 3c. These threads and an ' $\mathrm{O}$ ' ring groove will assist in realizing a leak proof connection between the sensor device and the pressure calibrator.

For a rigidly clamped diaphragm under uniform pressure (such as in the present case), the radial and tangential strains at the center of the diaphragm are almost identical. The radial strain decreases so rapidly that it becomes negative and reaches twice the center strain at the edge, as the radius increases. The tangential strain decreases from the center value to zero around the periphery of the diaphragm. The strain gauge pattern to be screen printed on the diaphragm is designed to benefit from this kind of strain distribution. Due to the fact that the tensile and compressive strains simultaneously exist, the sensing films are printed and connected in Wheatstone configuration in such a way that, the effects in four active arms become additive that provides a higher output. The thickness of the diaphragm is chosen so that it produces about 250 micro strains at a nominal pressure range (50 Bar). The present work makes the realization of graphite composite based piezoresistive printed sensors easy, by choosing suitable printing ink and graphite powder. However, the quality of the printed films is determined by the formulation of resistive dispersion. The mix proportions of printing ink and graphite powder are to be considered important to achieve optimum performance of the realized pressure sensors [15].

\subsection{Pressure transduction characterization}

In order to carry out the pressure transduction characterization of the fabricated devices, they are mounted on a dead weight hydraulic pressure calibration system. Figure 5 shows the equipment used to calibrate the pressure sensor. Some of the essential specifications of the calibrator are given in Table 3 .

The pressure sensor is connected to the pressure inlet adaptor (Fig. 6) of the calibrator. Figure 7 shows the setup used to calibrate the realized pressure sensor device.

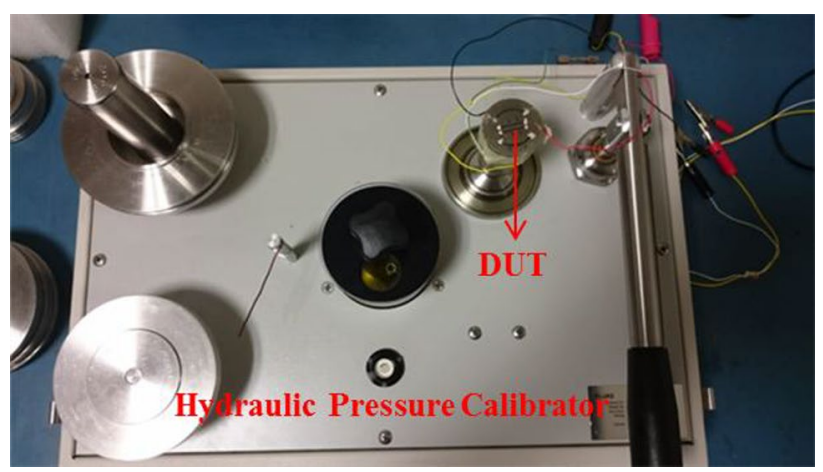

Fig. 5 Pressure sensor mounted on hydraulic pressure calibrator 
Table 3 Some of the essential specifications of pressure calibrator used

\begin{tabular}{ll}
\hline Make & FLUKE, phoenix, AZ, USA \\
Model number & P3125XT -BAR \\
Accuracy & $0.008 \%$ \\
Range & 1 to $1100 \mathrm{Bar}$ \\
Gravity & $9.80665 \mathrm{~m} / \mathrm{s}^{2}$
\end{tabular}

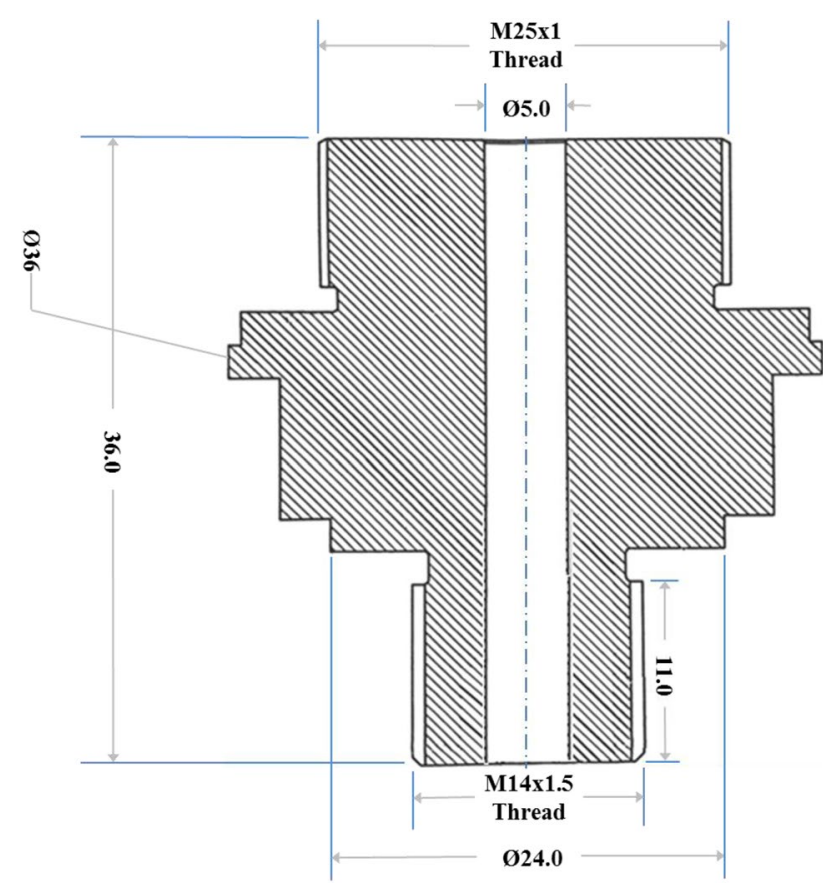

Fig. 6 Schematic of pressure inlet adaptor (all dimensions are in $\mathrm{mm}$ )

A known excitation voltage of $5 \mathrm{~V}$ is applied across the input of strain gauge Wheatstone bridge through the leads, using a DC voltage source. Leads from the bridge output are connected to a $61 / 2$ digit multimeter (Gwinstek, GDM-8261), for electrical readouts. A known pressure is applied to the sensor, using the dead weights provided in the calibration system. The pressure transduction technique can be portrayed as in Fig. 1b. The hydraulic input pressure causes a proportional deflection of sensing diaphragm in the upward direction. Upon depressurizing, it causes downward deflection of the diaphragm. The diaphragm deflection introduces structural deformations of the screen-printed, dispersion based, piezoresistive films, causing a change in bridge output voltage. Hence, it can be resolved that the pressure transduction can be realized by measuring a change in output voltage that directly proportional to the input pressure applied.

In our work, gauge factor of the pressure sensor is calculated based on stainless steel diaphragm deflections. The maximum applied pressure is 50 Bar which corresponds to 250 micro strains. The pressure is varied in cyclic manner (ascending and then descending) and, the output was noted in 5 equal intervals of input pressure. Along with noting the variation of bridge output voltage as well as measuring open circuit Wheatstone bridge's individual resistances, the relative change in resistance is also recorded.

\section{Results}

\subsection{Resistive dispersion-based pressure sensor characterization}

As a background work for the current study of resistive dispersion-based pressure sensors, experimentations were carried-out on graphite dispersion-based sensing film strain gauges. Their material and strain sensing characterizations are reported in Subramanya et al. [15]. During this work we found that, samples prepared using 75:25 mix proportion dispersion (ink: graphite, mixed by wt\%) yield the highest GF along with satisfactory performance parameters like linearity, repeatability, temperature coefficient of resistance (TCR) etc. Hence in the present work, we chose the dispersion of same mix proportion (75:25). Utilizing the observations and characterization results of the prior investigation, as a further experiment, the design and analysis of resistive dispersion-based pressure sensor device is reported in the present study. The experimental
Fig. 7 Pressure sensor calibration set-up

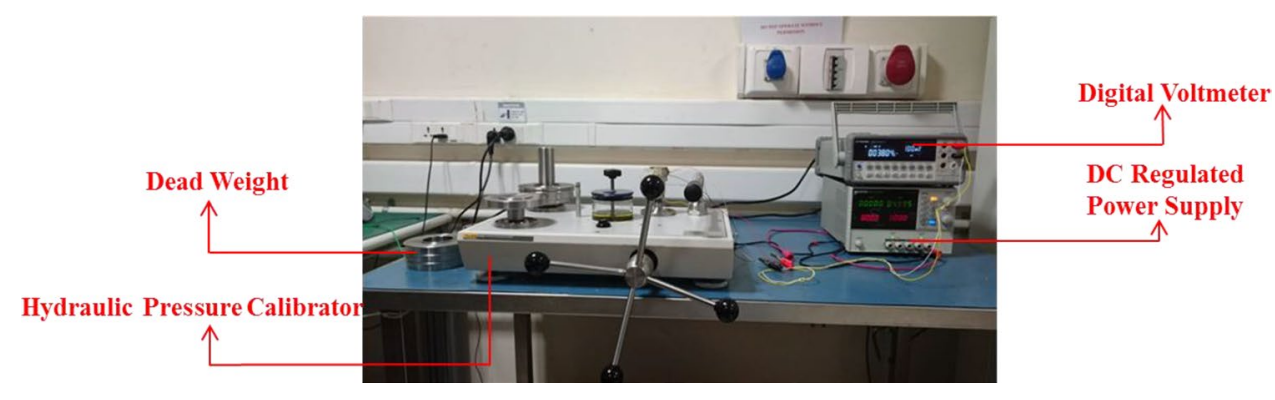


results pertaining to their pressure transduction characteristics are discussed further.

\subsubsection{Performance of pressure sensor}

Carbon/graphite inks turns out to be highly economical, comparing the prices of $1 \mathrm{~g}$ of silver and $1 \mathrm{~g}$ of graphite powder, the cost of conductive inks can be reduced by at-least 10 times when graphite is used instead of metallic fillers like silver. Considering the feature of tailoring the film resistance, it is hard to tune the value of film resistance when metallic inks are used whereas it can be easily accomplished by varying the amount of graphite filler in the sensing composite [16]. In our present study, the printed films display higher values of gauge resistance (in $\mathrm{k} \Omega$ ) in the unloaded state. This is advantageous due to the fact that, sensing films with higher gauge resistances exhibit a larger change in resistance with the applied pressure [15]. Also, the issues related to non-reproducibility and instability found in very thin and discontinuous films due to very large gauge resistances are overcome in the present approach, by making use of screen-printing technique to synthesize the sensing films. Thickness of screenprinted films varies in the range 13-27 $\mu \mathrm{m}$ [15].

As M-Bond 610 adhesive coating provides a strong insulation resistance, its choice turns out to be highly beneficial as an insulating layer on the diaphragm. In the present work, the insulation resistance is found to be about $800 \mathrm{M} \Omega$ at $50 \mathrm{~V} \mathrm{DC}$ which is quite adequate for the transducer operation, without causing any output degradation. The use of such coating materials for insulating the diaphragm makes the process cost effective by avoiding complex and expensive techniques such as multilayer oxide deposition using reactive electron beam, RF magnetron sputtering or ion beam methods. The present insulation technique is also viable for mass production. Moreover, the additional advantages of using the present methodology is that, pinhole free insulation layers can be realized and also, they exhibit a strong adhesion to the substrates.

The stability of screen-printed sensing films is indicated in Fig. 8. It shows the variation of resistance of individual films (denoted as RX, where ' $X$ ' corresponds to 1 st, 2 nd, 3 rd or 4th sensing films) in unloaded state, observed over 6 days. In the present study, stability investigation of the sensing films is carried out at ambient conditions. The device is subjected to room temperature, pressure and humidity conditions, with the diaphragm unpressurized. A variation of $\pm 3^{\circ} \mathrm{C}$ change in temperature with a negligible change in pressure and humidity are observed during these measurements. Considering the stability performance of the printed strain gauge film based pressure sensors, it is a well-established fact that the sensing composite formulation and film insulation techniques employed

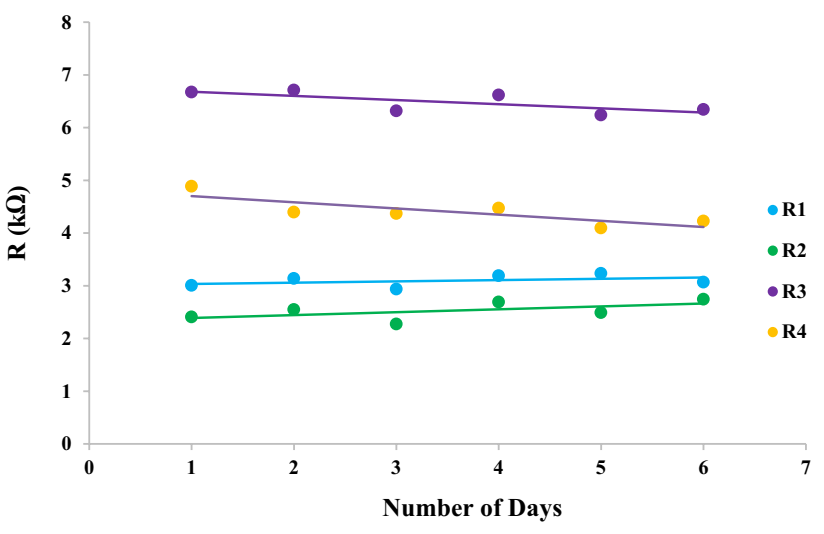

Fig. 8 Variation of resistance of individual films over time in unloaded state

to realize the strain gauge films have high influence on the stability characteristics [15]. In the current investigation, the sensing films are found to be stable at ambient conditions and the overall change in resistance with time, of all the printed piezoresistive films is found to be less than $2 \%$. This kind of stability feature is useful in achieving consistent and repeatable results for the pressure sensors which are implemented using printed strain gauge films. The stability, hysteresis response and repetitive nature of the graphite based pressure sensors observed in the present work are in agreement with the literature [10-12].

As part of the calibration of the realized pressure sensor device, zero offset value is adjusted close to $0 \mathrm{mV}$ (by balancing the Wheatstone bridge using an external resistance and potentiometer). The end loops and solder tabs are considered insensitive to strain because of their relatively very small cross-sectional area and low electrical resistance. Pressure cycles are carried out on the sensor by applying hydraulic pressure in the range from 0 to 50 Bar. The hydraulic input pressure is varied in steps of 10 Bar, both in ascending and descending modes. Figure 9a shows the variation of combined nonlinearity \& hysteresis characteristics of the films, across the trials. In the initial pressure cycles it is found to be about $1.167 \%$ FSO at 50 Bar. However, by observing the characteristics it can be perceived that the nonlinearity \& hysteretic response of the sensor improves with trials. Hence, the pressure cycling is continued till a lower and stable nonlinearity is achieved. Figure $9 \mathrm{~b}$ shows a comparison between the first and last pressure cycles. As can be observed in the figure, the nonlinearity \& hysteresis is found to be about $0.732 \%$ FSO in the last cycle. A very little change (less than $0.1 \%$ ) is observed in the further cycles. The low nonlinearity and hysteresis characteristics displayed by the sensing films in the current work support the fact that the printed films can possess excellent elastic characteristics. Moreover, 

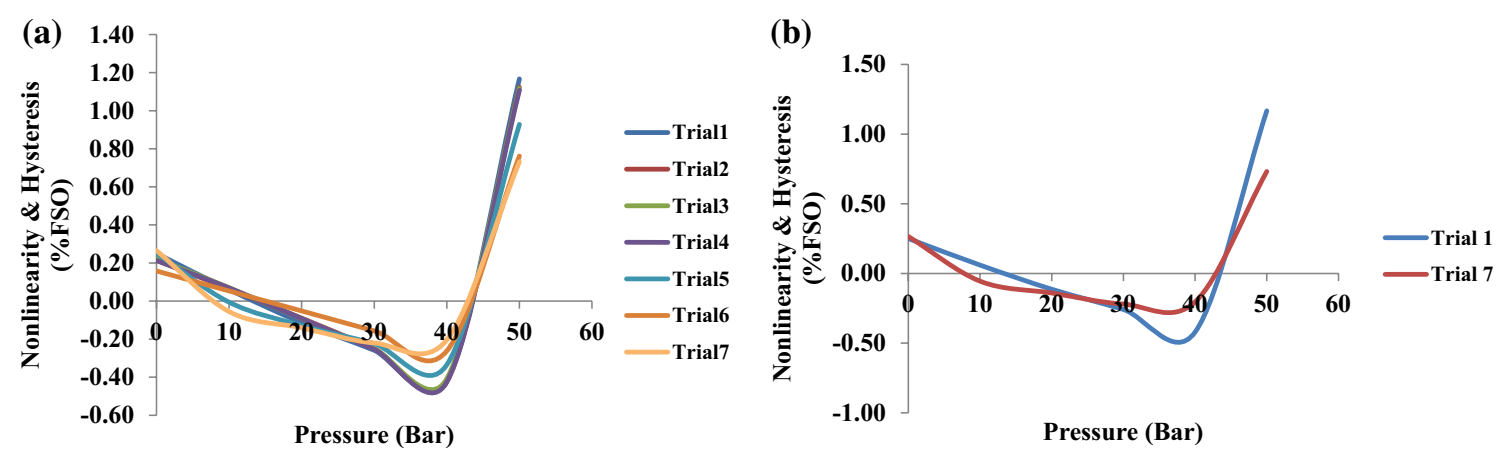

Fig. 9 Variation of combined nonlinearity \& hysteresis with applied input pressure a across trials, $\mathbf{b}$ trial 1 and 7 combined

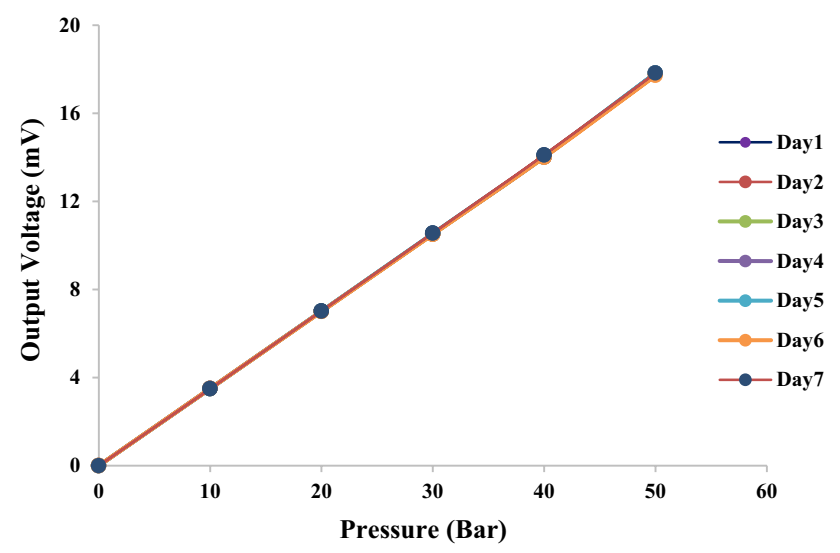

Fig. 10 Variation of bridge output voltage with applied input pressure

if this sensor is used between 0 and 45 Bar pressure, a non-linearity and hysteresis below $\pm 0.4 \%$ can be easily achieved (Fig. 9).

The bridge excitation voltage is set to $5 \mathrm{~V}$ so as to avoid excessive current and formation of hot spots within the printed films that may lead to unstable variation of bridge output voltage, due to higher excitation voltages. Figure 10 shows the pressure transduction response of the sensor in terms of variation of output voltage with applied input pressure. During the pressure cycling in the present work, the output voltage is found to be in the range from 0 to $17.83 \mathrm{mV}$, as shown in Fig. 10. It is evident from the plot that the sensor exhibits a linear response in the entire pressure range. Figure 11 shows the variation of sensitivity across the number of trials. In the plot it can be perceived that the sensitivity of the device is about $0.3565 \mathrm{mV} / \mathrm{bar}$ and it can be observed that, it remains consistent across the trials.

Figure 12 shows the variation of maximum combined nonlinearity \& hysteresis across seven number of pressure cycles. From the figure it can be clearly witnessed that, the maximum nonlinearity $\&$ hysteresis of the

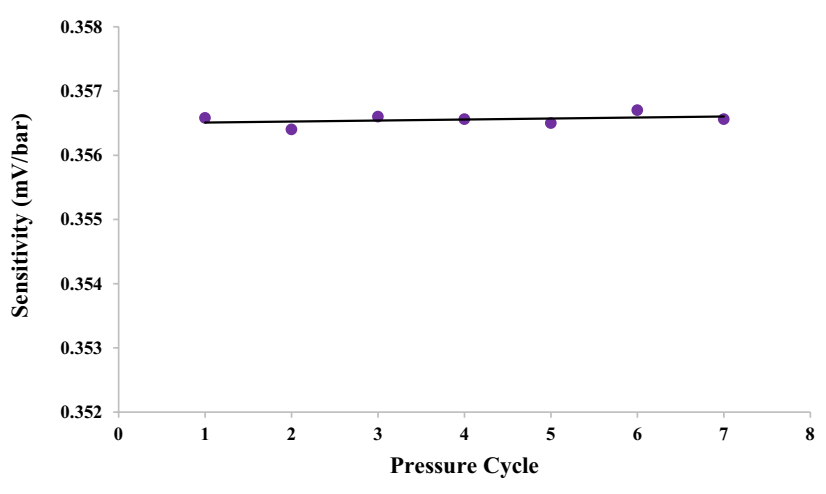

Fig. 11 Variation of sensitivity across trials

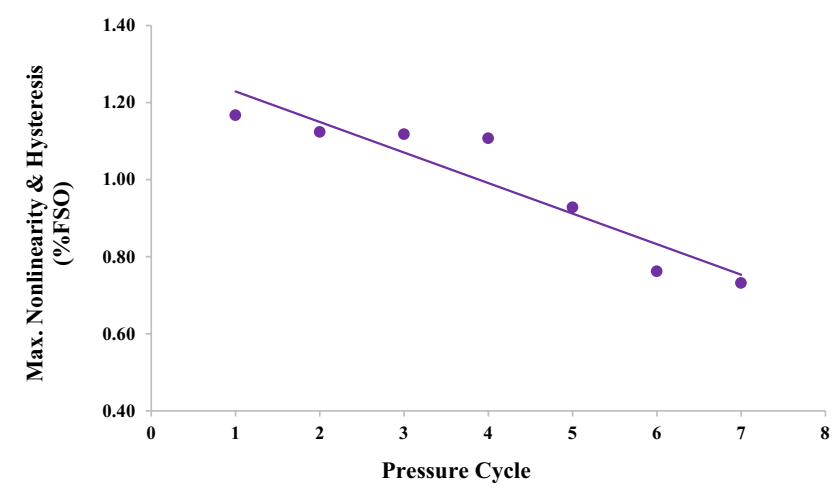

Fig. 12 Variation of maximum cobined nonlinearity \& hysteresis with number of pressure cycles

realized pressure sensor shows a decreasing trend with number of pressure cycles. It decreases from $1.167 \%$ FSO in cycle 1 to $0.732 \%$ FSO in cycle 7 . This reduction corresponds to a value of $62.43 \%$, indicating a remarkable improvement in the nonlinearity \& hysteresis response. The flexible nature of the printed films allowed free movement of fillers avoiding any brittle nature [10]. 
In the present work, the span, nonlinearity, hysteresis and sensitivity are calculated using method of least squares best fit straight line. The results obtained during the calibration are tabulated in Table 4. It can be observed from the results that the screen-printed pressure sensors provide reliable and robust strain readouts and their performance is at par with glued sensors [2].

The flexibility and robustness of the pressure sensors observed in the present study can be attributed to the various entities such as sensing composite materials, device substrate and operating conditions. Primarily when the strain sensing composite is synthesized using a combination of materials (as in the present case), the pressure sensing behavior of the device can be related to the synergic effects of all the materials in the composite. This fact is supported by numerous demonstrations reported in the literature $[10,11,13,15]$.

\section{Merits of the present study}

The design and characterization of resistive dispersion based pressure sensor is successfully carried-out in the present work. The merits of the current investigation can be realized by considering the materials set used to synthesize the sensing composite and, the technique used to form piezoresistive films on the pressure sensor diaphragm. A low cost, robust, resistive dispersion, synthesized using the graphite powder PVC resin composite is printed on the stainless steel diaphragm. The usage of this composite, instead of other carbon based and metallic fillers, makes the current approach more economical. Screen printing technique is employed in the current work to implement strain gauges of the pressure sensor device. This technique can be easily incorporated in ambient operating conditions (without demanding for cleanroom environment, high/low pressure or temperature conditions, vacuum etc.) that lead to easy fabrication of the pressure sensor devices for mass production with cost reduction.

Table 4 The span, nonlinearity and hysteresis data obtained during the calibration

\begin{tabular}{lll}
\hline Trial number & Span $(\mathrm{mV})$ & $\begin{array}{l}\text { Max. nonlinearity \& } \\
\text { hysteresis (\% FSO) }\end{array}$ \\
\hline 1 & 17.828 & 1.167 \\
2 & 17.830 & 1.126 \\
3 & 17.825 & 1.118 \\
4 & 17.828 & 1.101 \\
5 & 17.830 & 0.928 \\
6 & 17.722 & 0.762 \\
7 & 17.821 & 0.732 \\
\hline
\end{tabular}

Screen printing technology also provides a variety of substrate choices such as glass, acrylic, poly dimethyl siloxane (PDMS), polyethylene terephthalate (PET, such as Mylar), polyimide (PI, such as Kapton), paper, metal, metal alloys, 3D printable structures etc. The strain sensing films can be easily produced by controlled hot air drying the printed patterns. Exposing the printed composite to special light sources like UV is not required to form strain sensing films. A strong adhesion between the composite and diaphragm is achieved by the formulation of strain sensing composite. Special formulations to address the adhesion issues that involve choosing the resins, adhesives, fillers etc. are not necessary. They are handled by the physical and chemical properties of the letterpress ink used in the present work. The wetness of the ink offers ease of printing and it also holds the graphite particles intact. Moreover, since nontoxic materials are used to synthesize the sensing composite, the devices are safely disposable also. This feature provides possibilities of employing these devices in biomedical applications [10], flexible sensors, tattoo sensors etc. The strain sensing composite formulation techniques to make them suitable for pressure sensing applications and the purpose of utilizing the screen printing technique in the present work is also elaborated in Subramanyaet al. [15].

\section{Conclusion}

In the present work, design and characterization of resistive dispersion based pressure sensor devices is explored. Since high GF was observed during characterization of the printed piezoresistive films in our previous study, implementation of the pressure sensor devices using these films is attempted. Films turn out to be ideal for transducers because of their higher gauge resistance and gauge factor. In the current investigation, pre-formulated resistive dispersions synthesized using graphite powder and PVC resin based composite is patterned over stainless steel diaphragm of a gauge pressure sensor using screen printing technique. The dispersion can be easily printed on flexible substrates such as PDMS, PET or also clothes as wearable electronics for motion (movement) detection [13]. The realized devices were found to be stable at ambient conditions, robust and exhibited a consistent high performance.

The sensitivity of the graphite-polymer based sensors is higher as compared to that of the metallic fillers such as silver [2]. In the present work, the sensitivity is found to be $0.357 \mathrm{mV} / \mathrm{bar}$. It is higher than those sensors, obtained using metallic nanoparticles [13]. The maximum non-linearity and hysteresis are calculated to be less than $0.8 \%$ FSO for a range of 0 to 50 Bar. Moreover, non-linearity and hysteresis is $\pm 0.45 \% \mathrm{FSO}$ for a range below 45 Bar. A fairly 
satisfactory sensitivity, linearity, hysteresis and stability are accomplished at a low cost.

For measuring the strains in multiple directions, the films can be easily printed in various directions and these sensors can be deployed in structural health monitoring applications. Robotic applications may utilize these sensors to detect bending and movement. From the fabrication point of view, safe materials are being used. Hence, it may be also used in some of the biomedical applications which are already attempted by authors like Nag et al. [10, 17], Wu et al. [18]. Since he sensitivity is high enough, they may be used in artificial skin (e-skin) applications to sense minute touch on the body and other wearable sensors. There is also a possibility of using these sensors in prosthetic limbs for tactile sensing. It may be a replacement for commercialized tactile sensors implemented using materials like Dacron and Kevlar.

\section{Compliance with ethical standards}

Conflict of interest The authors declare that they have no conflict of interest.

\section{References}

1. Reynolds JK et al (2000) Packaging a piezoresistive pressure sensor to measure low absolute pressures over a wide sub-zero temperature range. Sens Actuators 83:142-149

2. Kulha $P$ et al (2017)Temperature dependence of gauge factor of printed piezoresistive layers embedded in organic coatings. In: Proceedings, vol 1.p 618

3. Bae SH, Lee Y, Sharma BK, Lee HJ, Kim JH, Ahn JH (2013) Graphene-based transparent strain sensor. Carbon 51:236-242. https://doi.org/10.1016/j.carbon.2012.08.048

4. Monty CN, Wujcik EK, Blasdel NJ (2017) Flexible electrode for detecting changes in temperature, humidity, and sodium ion concentration in sweat. US patent $9,603,560$

5. Casiraghi $C$ et al (2018) Inkjet printed 2D-crystal based strain gauges on paper. Carbon 129:462-467
6. Jing Z, Guang-Yu Z, Dong-Xia S (2013) Review of graphenebased strain sensors. Chin Phys B 22:05770

7. Zheng X, Chen X, Kim JK, Lee DW, Li X (2013) Measurement of the gauge factor of few-layer grapheme. J Micro Nanolithogr MEMS MOEMS 12(1):013009. https://doi.org/10.1117/1. JMM.12.1.013009

8. Serra N et al (2012) Piezoresistive effect in epoxy-graphite composites. Sens Actuators A 186:198-202

9. Dinh T et al (2015) Graphite on paper as material for sensitive thermoresistive sensors. J Mater Chem C R Soc Chem

10. Nag A, Feng S, Mukhopadhyay SC, Kosel J, Inglis D (2018) 3D printed mould-based graphite/PDMS sensor for low-force applications. Sens. Actuators A Phys 280:525-534

11. Gupta AA, Bolduc A, Izquierdo R (2016) Aerosol jet printing for printed electronics rapid prototyping, 978-1-4799-5341-7/16. IEEE

12. Bona M, Serpelloni M, Sardini E, Lombardo CO, Andò B (2017) Telemetric technique for wireless strain measurement from an inkjet-printed resistive sensor. IEEE Trans Instrum Meas 66:583-591

13. Joshi P, Santhanam V (2018) Strain-sensitive inkjet-printed nanoparticle films on flexible substrates. Mech Sens IEEE Sens Lett 2(1):1-4

14. Enser H, Sell JK, Hilber W, Jakoby B (2017) Hysteresis and material effects of printed strain gauges embedded in organic coatings. In: Eurosensors PARIS Proceedings, vol 1, p 624. https://doi. org/10.3390/proceedings 1040624

15. Subramanya SB et al (2019) Study and tailoring of screenprinted resistive films for disposable strain gauges. Sens Actuators A 295:380-395

16. Bhore SS (2013) Formulation and evaluation of resistive inks for applications in printed electronics, Master's Theses, Paper 424

17. Nag A, Afasrimanesh N, Feng S, Mukhopadhyay SC (2018) Strain induced graphite/PDMS sensors for biomedical applications. Sens Actuators A Phys 271:257-269

18. Wu Y, Karakurt I, Beker L, Kubota Y, Xu R, Ho KY, Zhao S, Zhong J, Zhang M, Wang X, Lin L (2018) Piezoresistive stretchable strain sensors with human machine interface demonstrations. Sens Actuators A Phys 279:46-52

Publisher's Note Springer Nature remains neutral with regard to jurisdictional claims in published maps and institutional affiliations. 